Research Article

\title{
Liquid Force and Rupture Distance between Two Particles
}

\author{
Cheng Pu $\mathbb{D}$, Fengyin Liu, and Shaohan Wang \\ Dept. of Geotechnical Engineering, Xi'an University of Technology, Xi'an, China \\ Correspondence should be addressed to Cheng Pu; 1909488416@qq.com
}

Received 3 June 2021; Revised 3 August 2021; Accepted 6 August 2021; Published 19 August 2021

Academic Editor: Tetsu Yonezawa

Copyright (c) 2021 Cheng Pu et al. This is an open access article distributed under the Creative Commons Attribution License, which permits unrestricted use, distribution, and reproduction in any medium, provided the original work is properly cited.

\begin{abstract}
The study of liquid force has a special meaning to industrial manufacturing. By taking the liquid bridges between equal and unequal particles as objects, the liquid force-displacement curves were measured and recorded by using a novel Nano UTM T150 tensile system. The influences of diameter, diameter ratio, liquid volume, and the surface tension on the liquid force-distance curve, the maximum liquid force, and rupture distance were compared and sorted. The results show that the maximum liquid force and rupture distance both increase with the increase in liquid volume, particle diameter, diameter ratio, and surface tension. The diameter plays a decisive role in determining the value of the maximum liquid force compared with surface tension and liquid volume, which only influence the force value in a local range. The rupture distance shows a positive correlation with liquid volume and surface tension and a negative correlation with the diameter or diameter ratio. The maximum liquid force between unequal particles is about half of the sum of the force between the equal spheres of larger and smaller size in that system.
\end{abstract}

\section{Introduction}

Liquid force, which is commonly encountered in nature, has been widely applied in industries such as crystallization and agglomeration, deinking of recycled paper, and removal of heavy metals from wastewater since the late 1960s. The study to quantify and estimate the liquid force has a positive effect on industrial manufacturing.

The pioneering study of liquid force can be traced back to the field of surface science, which was started by Hanines and Fishers [1], who studied the liquid bridge formed between two equal-sized spheres. Afterward, researchers studied the liquid force mainly via numerical simulation and experimental investigation. In the field of numerical simulation, Gillespie and Settineri [2] and Clark et al. [3] assumed the profile of liquid to be circular to find a simple and effective numerical computation. Mehrotra and Sastry [4] conducted a comprehensive review of the pendular liquid bridges research up to 1980 . Assuming the particles with thermodynamic equilibrium and the bridge maintain a constant mean-curvature and contact angle, De Bisschop and Rigole [5] and Lian et al. [6] theoretically studied the variation of liquid force between two smooth spheres during stretching. Mathewson [7] and Washino et al. [8] investigated the effect of viscous forces during the separation of a sphere and plate and the wet granular flowing process, respectively. Molenkamp and Nazemi [9] used a dimensionless method to investigate the relationship between the value of force and the solid-liquid angle, sphere radius, and surface roughness. Pirooz et al. [10] established a model to study the force and rupture distance of liquid bridges between two unequal spheres. Chen et al. [11] analyzed the development of liquid force between two spheres and a sphere-plate system. Rossetti and Simons [12] studied the rupture energy during the spherical stretching process. When the volume of a liquid bridge is large, the effect of gravity cannot be neglected; Mazzone et al. [13] and Farmer and Bird [14] studied the influence of gravity on liquid force and liquid profile while analyzing the condition of nonaxisymmetric bridge occurrence when gravity is considered from the view of theoretical derivation and numerical simulation. Zhang et al. [15] instigated the influences such as mineral type, water volume, and initial distance on the mechanical behavior of the liquid bridge between clay 
particles in Nanoscale. Pitois et al. [16, 17] proposed a common model that expresses liquid force as the sum of capillary force and viscous force.

In terms of the experimental study, early researchers measured liquid force mainly by differential balance and high-precision cantilever. Willett et al. [18] measured the liquid force between two equal and unequal synthetic sapphire particles by using a differential balance. Rossetti et al. [19] indirectly measured liquid force between a pair of equal particles by the deformation of a high-precision cantilever. With the improvement of testing methods, rigid testing machines have been widely used in liquid bridge tensile tests. Considering the evolution of the liquid bridge during the evaporation cannot be ignored [20], and nonvolatile liquids, such as glycerol, were used to conform to the constant volume condition. Bozkurt et al. [21] studied the influence of invasive angle on pendular liquid bridges between equal spheres. Pu et al. [22] measured the capillary force of liquids and the rupture distance between two moving spheres through separation. For systems more than two particles, the effective packing fraction of the particles has a significant impact on the force required to rupture such a bridge [23]. Lievano et al. [23] and Wang et al. [24] measured the development of liquid force within three particles. Working with small liquid volumes between two minimal glass spheres of $20-50 \mu \mathrm{m}$ in diameter, the liquid force was measured using a digital instrument AFM, and the experimental results were found to agree well with the theoretical results [25].

Research on the liquid force has made great progress, and the former results show that the value of liquid force is closely related to the liquid volume, spheres radius, the distance between particles, the surface tension of the liquid, and the contact angle. However, the magnitude of these effecting factors has not been compared yet. In this article, the influences of particle size, liquid volume, and surface tension on the value of liquid force and rupture distance were compared and sorted.

\section{Experimental}

2.1. Testing Instrument. The Nano UTM T150 tensile system, which is a novel apparatus manufactured by the American Keysight Company, was used for testing. The T150 system has been designed to be a universal testing system, which contains a rigid shell, vibration reduction table, data acquisition control (DAC) unit, and micropositioner. The rigid shell can minimize the fluctuation caused by the sudden release of stress when the liquid bridge breaks. The vibration reduction table can eliminate the influence of noise and vibration on experimental results. The data acquisition unit, which connects to the computer as the interface between the software and the T150 system, is housed in the electronics cabinet. The micropositioner, which is located at the bottom of the crosshead, allows the proper alignment of samples by moving grip in the plane. The main mechanical parameters of the Nano UTM T150 tensile system are shown in Table 1.

The schematic diagram of the testing structure is shown in Figure 1. Two high-precision microscopic CCD cameras were connected to a computer and placed perpendicular to each other in front and to the left of the testing machine to make sure the upper and lower particles were on the same axis.

2.2. Material. Glycerol and ethylene glycol were used to form the liquid bridge between two sphere particles due to their low evaporation and different surface tension. The physical parameters of these two kinds of liquid at $20^{\circ} \mathrm{C}$ are shown in Table 2. According to the results of Bozkurt et al. [21], the viscous force has a considerable effect on the force value only when the separation rate is higher than $4 \times 10^{3} \mathrm{~m} / \mathrm{s}$. The experiment in this study was conducted under static conditions; thus, the difference of viscosity coefficient can be neglected. Three different sizes of spheres as $2.5 \mathrm{~mm}, 4 \mathrm{~mm}$, and $5 \mathrm{~mm}$ in diameter and two kinds of liquid were selected for the experiment.

Spheres made of silicon dioxide with a smooth surface (roughness $\mathrm{Ra}=60 \mathrm{~nm}$ ) are selected for the experiment, and all particles in this article are supported by Xi'an Northwest Photoelectric Instruments Plant. The silanol groups on the surface of particles can be divided into isolated silicon hydroxyl groups, adjacent silicon hydroxyl groups, and auxiliary silicon hydroxyl groups. The concentration of silicon hydroxyl groups equals $4.12 \mathrm{OH} / \mathrm{nm}^{2}$. Measuring the critical surface tensions of the particles through the Zisman method, the critical surface tensions of the particles equal $43.6 \mathrm{dyn} / \mathrm{cm}$. The contact angles of liquid on the particle surfaces are shown in Table 3.

2.3. Test Procedure. The particles were rinsed with ethyl alcohol and deionized water to remove dust and eliminate electrostatic forces on the particle surface before experiment. Two particles were glued with a low-temperature melting glue. The upper particle was glued to the rigid steel, which could be moved up and down at a constant rate, and the lower was glued to the NMAT extension head. Then, the chosen liquid was placed on the top of the bottom particle by means of a microsyringe to create a bridge of known volume between the spheres pairs while the particles were separated. Several accommodation cycles of approach and separation were performed before starting the measurements to obtain a perfect axisymmetric bridge. Finally, the upper particle was moved upwards at a constant speed of $100 \mu \mathrm{m} / \mathrm{s}$ while the lower remained immobile.

The glycerol was used to form the liquid bridge firstly, and the liquid bridge tensile tests between equal particles were conducted by measuring the liquid force between two $2.5 \mathrm{~mm}$ particles, two $4 \mathrm{~mm}$ particles, and two $5 \mathrm{~mm}$ particles. After that, maintaining the lower particle size constant at $5 \mathrm{~mm}$, the upper particle was successively replaced with $2.5 \mathrm{~mm}$ and $4 \mathrm{~mm}$ particles. Finally, the glycerol was replaced with ethylene glycol to form the liquid bridge between two $2.5 \mathrm{~mm}$ particles. The ratio of the upper particle diameter to the lower particle diameter was defined as the diameter ratio $n$. The test conditions are shown in Table 4. The liquid volume was set as $0.1,0.2,0.5,1.0$, and $1.5 \mu \mathrm{L}$. 
TABLE 1: The main mechanical parameters of the Nano UTM T150.

\begin{tabular}{lcccc}
\hline Maximum load $(\mathrm{mN})$ & Load resolution $(\mathrm{nN})$ & Maximum displacement $(\mathrm{mm})$ & Displacement resolution $(\mathrm{nm})$ & Stretching rate \\
\hline 500 & 50 & 200 & 35 & $0.5 \mu \mathrm{m} / \mathrm{s}-5 \mathrm{~mm} / \mathrm{s}$ \\
\hline
\end{tabular}

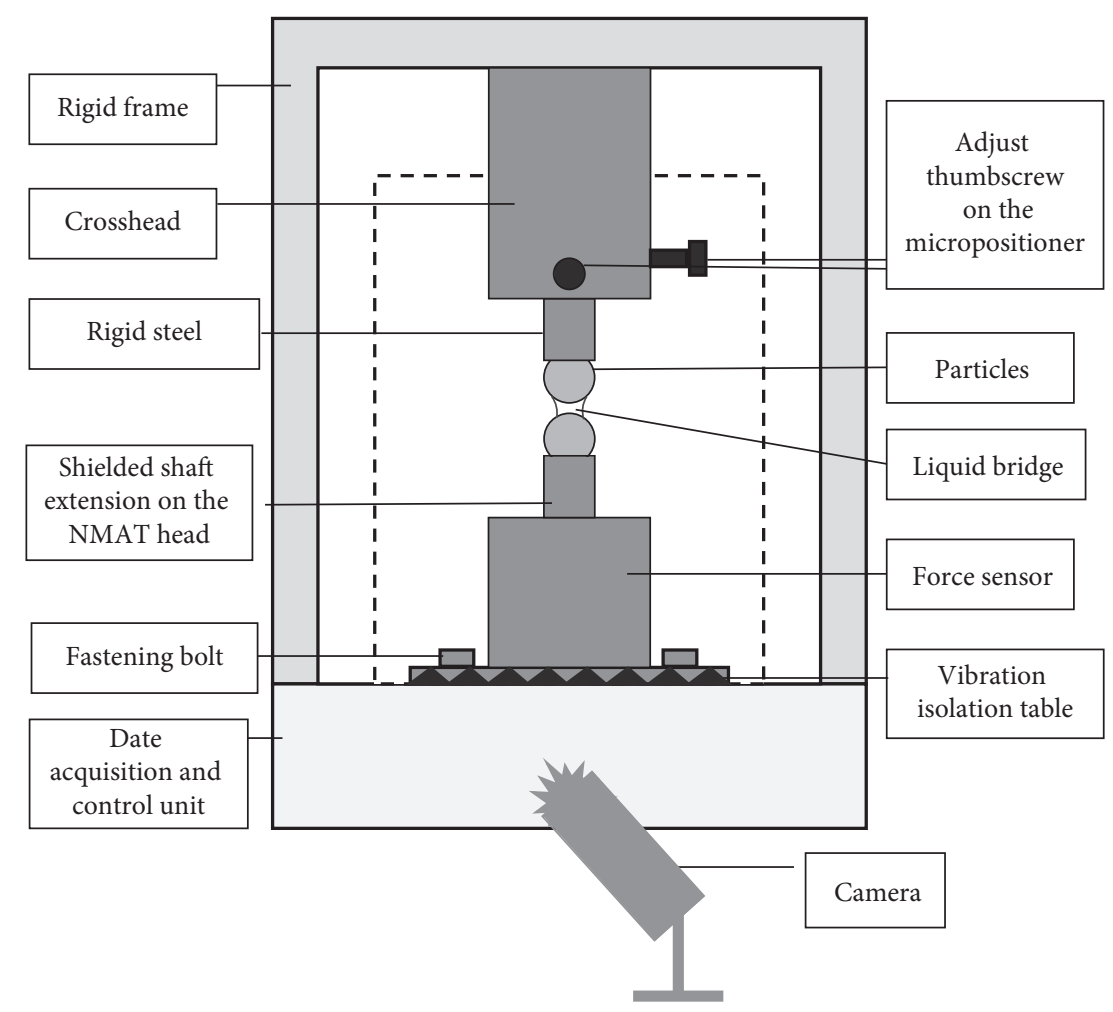

FIgURE 1: Schematic of the UTM T150 system experimental apparatus.

\section{Results and Discussion}

3.1. Correlation Theory. Figure 2 shows the stress state in the neck of the liquid bridge. As shown, there exists force $F^{\psi}$ produced by matric suction within the liquid bridge and force $F^{\sigma}$ produced by surface tension at the liquid-gas interface [26]. The liquid force can be regarded as a sum of the $F^{\psi}$ and $F^{\sigma}$, as shown in equation (1), and $F^{\psi}$ and $F^{\sigma}$ were calculated as equations (2) and (3).

$$
\begin{gathered}
F_{\text {liq }}=F^{\sigma}+F^{\psi}, \\
F^{\psi}=\pi r_{2}^{2} \psi, \\
F^{\sigma}=2 \pi r_{2} \sigma .
\end{gathered}
$$

The matric suction $\Psi$ at the gas-liquid interface can be expressed as equation (4) according to the Young-Laplace equation.

$$
\psi=\sigma\left(\frac{1}{r_{1}}-\frac{1}{r_{2}}\right)
$$

in which $r_{1}$ is the outer radius of the liquid bridge, $r_{2}$ is the radius of the neck of the liquid bridge, as shown in Figure 2, and $\sigma$ is the surface tension.
During the stretching process, the neck radius of the bridge and the solid-liquid contact angle decreased continuously, while the height of the liquid bridge and the outer radius increased continuously. Thus, $F^{\sigma}$ is always positive and can be regarded as an attractive force. $F^{\psi}$ is positive when $r_{1}$ is smaller than $r_{2}$ and negative when $r_{1}$ comes to larger than $r_{2}$ and can be regarded as attractive force and repulsive force, respectively.

3.2. Liquid Force-Displacement Curve. The results of the tensile tests are presented in Figure 3. It can be seen from Figure 3 that the liquid force increases with the increasing separation distance at first until it reaches a peak, at which point the force decreases with the increasing distance until it finally drops to zero. According to this, the curves can be divided into an ascent stage, a descent stage, and a rupture stage. It should be noted that, in the first stage, the maximum force appears to occur at a small but nonzero-separation distance $[13,21,25,27]$, while some previous research studies have shown that the maximum force occurs in the zero-separation distance [16-18].

Although this discrepancy was observed by many researchers, no one has discussed this phenomenon systematically. From the point of our view, this 
TABLe 2: Physical and mechanical parameters of glycerol and ethylene glycol $\left(20^{\circ} \mathrm{C}\right)$.

\begin{tabular}{lccc}
\hline Liquid & Density $\left(\mathrm{g} / \mathrm{cm}^{3}\right)$ & Surface tension $(\mathrm{N} / \mathrm{m})$ & Viscosity $(\mathrm{Pa} \mathrm{s})$ \\
\hline Glycerol & 1.26 & 0.063 & 0.15 \\
Ethylene glycol & 1.11 & 0.048 & 0.015 \\
\hline
\end{tabular}

TABLE 3: Contact angle of liquid on the particle surfaces $\left(20^{\circ} \mathrm{C}\right)$.

\begin{tabular}{lccc}
\hline Liquid & Static $\left({ }^{\circ}\right)$ & Advancing & Receding \\
\hline Glycerol & 32.1 & 38.0 & 28.5 \\
Ethylene glycol & 25.8 & 29.4 & 21.8 \\
\hline
\end{tabular}

TABLE 4: Test conditions.

Particle diameter $(\mathrm{mm})$

Equal particles $4: 4,5: 5$

Unequal particles $2.5: 5(n=0.5), 4: 5(n=0.8)$

Equal particles $2.5: 2.5$
Types of liquid

Glycerol

Glycerol and ethylene glycol discrepancy may be attributed to the difference of the initial conditions for different liquid tensile testing methods. Traditionally, there are two main methods used to conduct a liquid bridge tensile test. The method utilizing the high-precision cantilever, for example, starts at a critical state, where no compressive stress is produced in the deflection. Under this circumstance, the experiment starts at equilibrium though the separation distance may not be zero. With the stiff-testing machines that are widely used in this type of test, however, the experiment starts at a state in which the separation distance is absolute zero although there may be compressive stress at the top of the bottom particles. In the descent stage, the force $F^{\sigma}$ decreases continually, and the force $F^{\psi}$ firstly reduced in a positive direction then increased in a negative direction. In this stage, $r_{1}$ is always larger than $r_{2}$, and there exist both a repulsive force and an attractive force in the liquid bridge. Based on the theory in the former section, by substituting equation (4) back into equation (3), equation (5) is obtained, where

$$
F^{\psi}=\pi\left(\frac{r_{2}^{2}}{r_{1}}-r_{2}\right) \sigma
$$

For equation (5), given $r_{1}>r_{2}, \pi\left(r_{2}^{2} / r_{1}-r_{2}\right)<0$. The absolute values of equations (2) and (5) were compared, as shown in equation (6); because of $r_{1}>r_{2}$, the ratio of equation (2) to equation (5) is greater than 1 .

$$
\frac{\left|2 \pi r_{2}\right|}{\left|\pi\left(r_{2}^{2} / r_{1}-r_{2}\right)\right|}=\frac{\left|2 r_{1}\right|}{\left|r_{2}-r_{1}\right|}>1 \text {. }
$$

Therefore, although in this stage, the attractive force decreases rapidly, and the repulsive force increases, the attractive force is always higher than the repulsive force, and the attractive force is still predominant in the bridge.

When the neck of the bridge becomes the narrowest, the attractive force decreases to a minimum, the repulsive force reaches its maximum, and the curve reaches the third stage. In this stage, the bridge can no longer withstand any additional elongation, and it breaks abruptly. The remaining part of the curve represents the weight of the droplets remaining on the lower ball.

3.3. Maximum Liquid Force. The maximum liquid forces under different test conditions are shown in Figure 4 in which Figures 4(a) and 4(b) show the maximum liquid force between equal and unequal particles, respectively.

It can be seen from Figures 4(a) and 4(b) that the liquid force increases with the increase in particle diameter, diameter ratio, liquid volume, and surface tension. When the liquid volume increases from $0.1 \mu \mathrm{L}$ to $0.5 \mu \mathrm{L}$, the liquid force has a relatively high growth rate. When the volume changes from $0.5 \mu \mathrm{L}$ to $1.5 \mu \mathrm{L}$, the rate begins to decrease. The volume at which the liquid force growth rate transitions from high to low is defined as the critical volume. When the liquid volume is under this volume, the liquid force increases significantly with increasing volume; when the liquid volume is higher than this volume, the liquid force increases slowly with increasing volume. It can be inferred that the liquid force will not always increase with the liquid volume; once the liquid volume reaches a certain value, the maximum force will remain stable.

Surface tension also has an effect on the maximum liquid force. When the liquid volume between particles is the same, the larger the surface tension is, the higher the maximum liquid force will gain.

Moreover, the diameter and diameter ratio have a much bigger impact than the liquid volume and surface tension on the liquid force, and the liquid force in different sized particles distributes in various regions and shows a zonal distribution trend. When the diameter is $2.5 \mathrm{~mm}$, the liquid force is between $0.18 \mathrm{mN}$ and $0.24 \mathrm{mN}$; when the diameter is $4 \mathrm{~mm}$, the liquid force is between $0.33 \mathrm{mN}$ and $0.40 \mathrm{mN}$; and when the diameter is $5 \mathrm{~mm}$, the liquid force is between $0.49 \mathrm{mN}$ and $0.58 \mathrm{mN}$. For the unequal systems, when $n=0.5$, the liquid force is between $0.35 \mathrm{mN}$ and $0.38 \mathrm{mN}$; when $n=0.8$, the liquid force is between $0.42 \mathrm{mN}$ and $0.47 \mathrm{mN}$; and when $n=1$ (equal particles with a diameter of $5 \mathrm{~mm}$ ), the liquid force is between $0.49 \mathrm{mN}$ and $0.58 \mathrm{mN}$. 


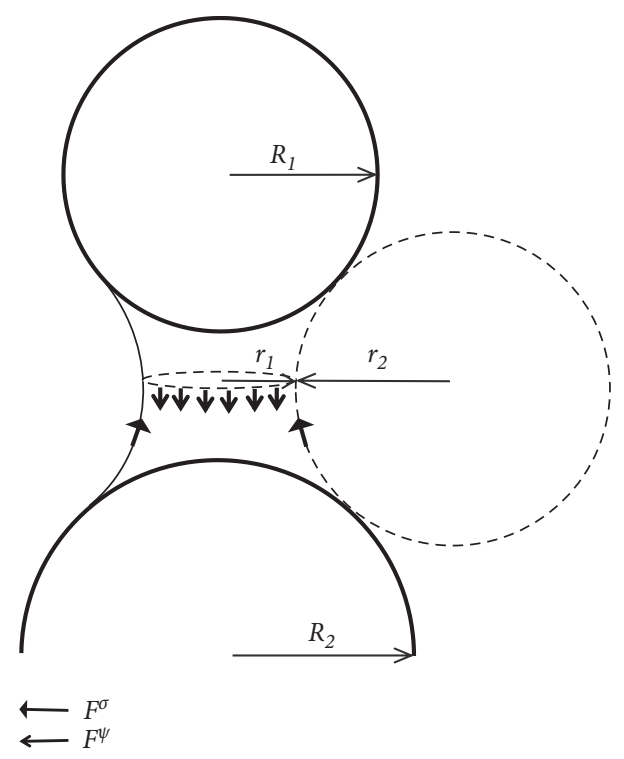

Figure 2: The force in the neck of a liquid bridge.

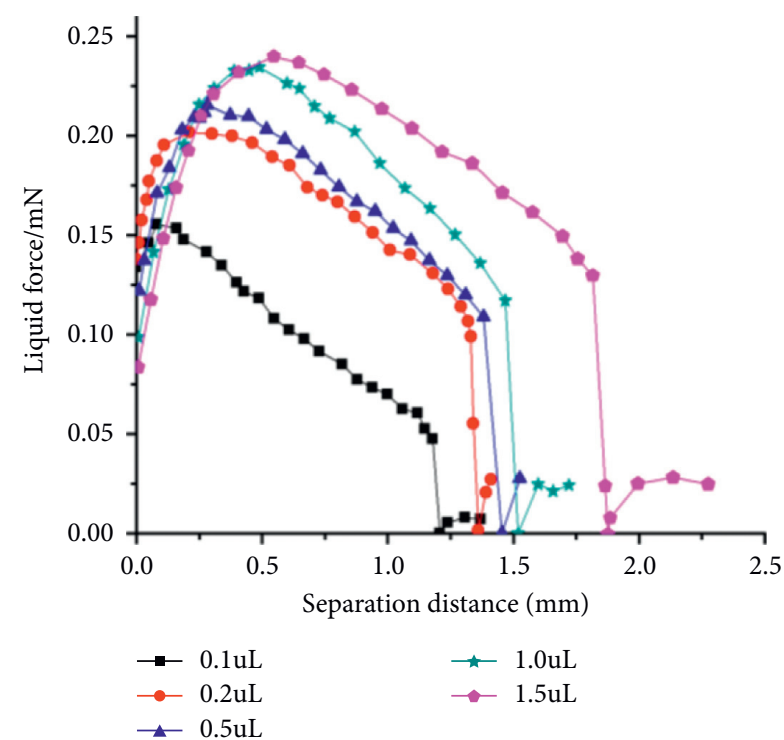

(a)

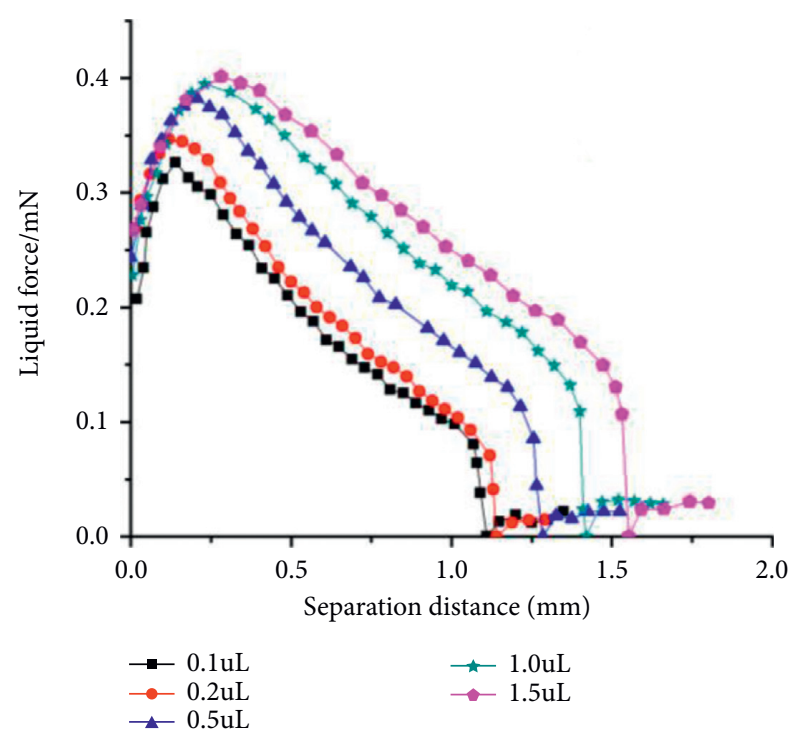

(b)

Figure 3: Continued. 


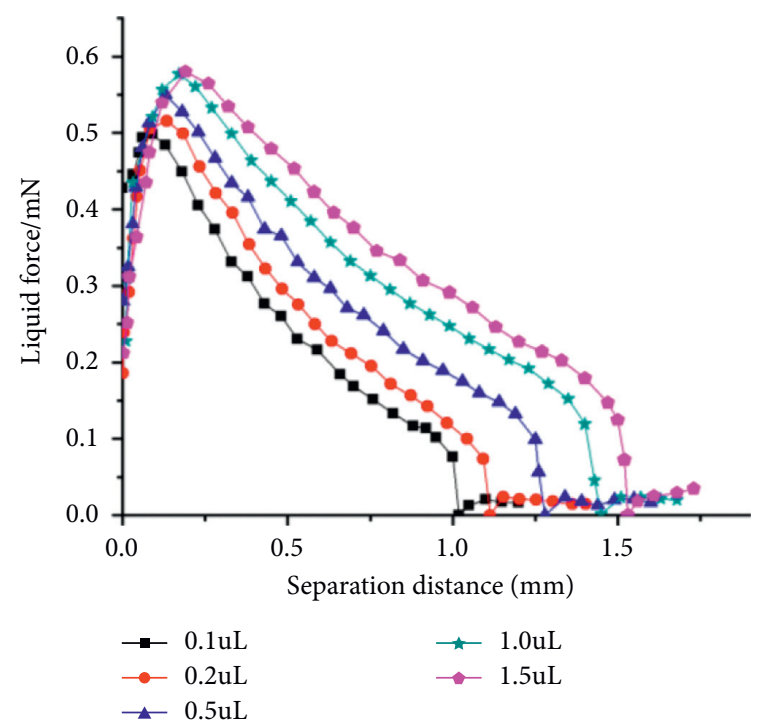

(c)

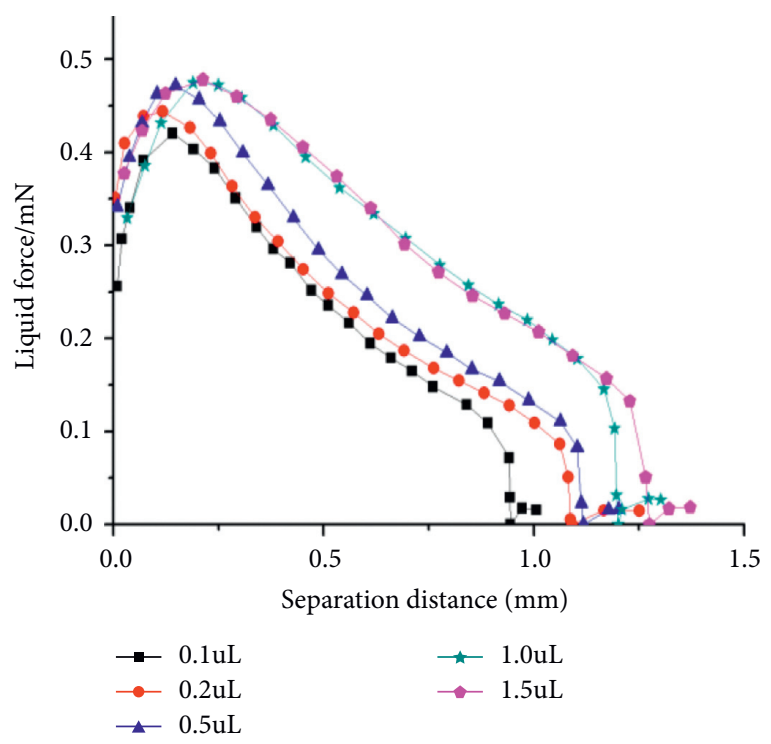

(e)

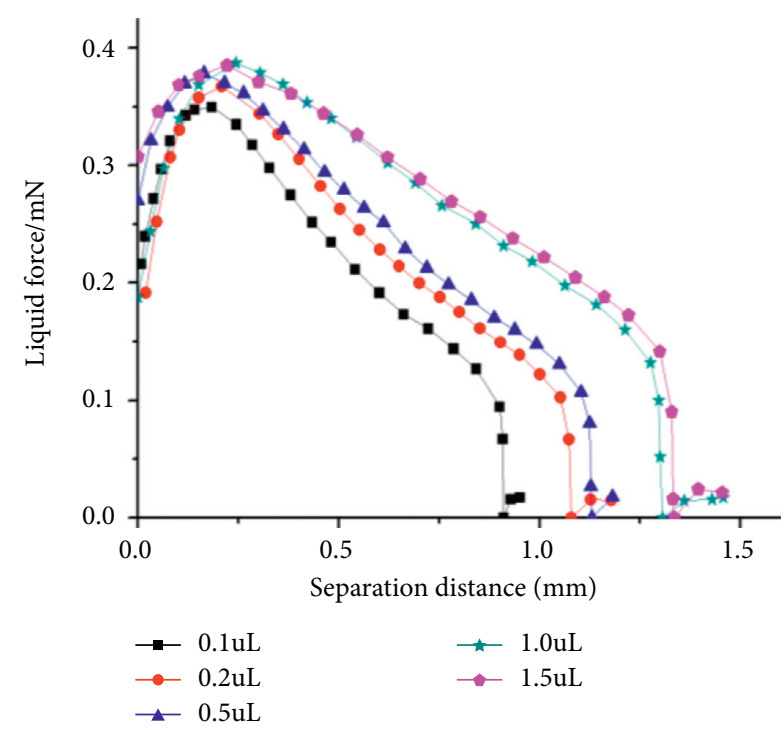

(d)

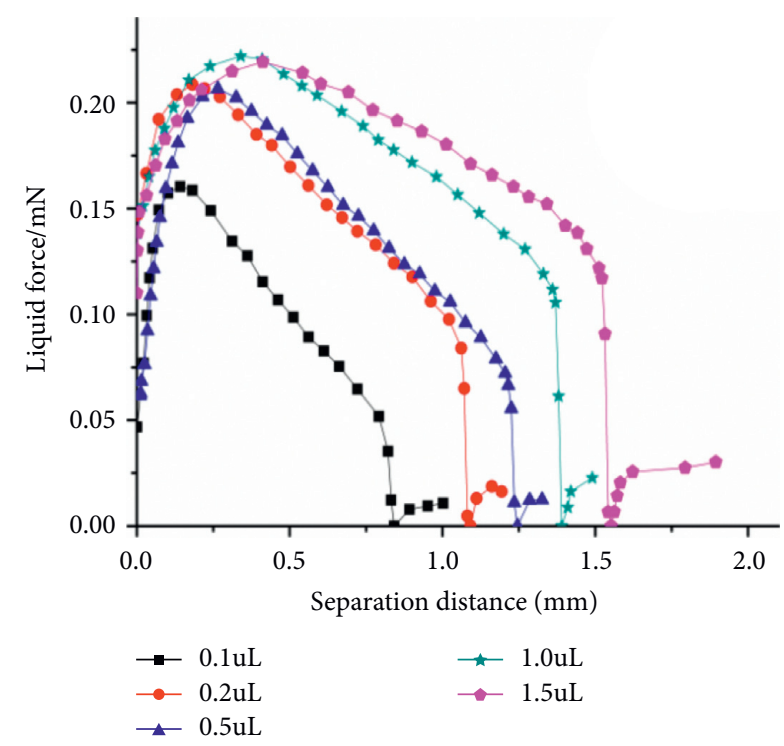

(f)

Figure 3: The liquid force-separation distance curve under different test conditions: (a) $2.5 \mathrm{~mm}: 2.5 \mathrm{~mm}$ made by glycerol; (b) $4 \mathrm{~mm}: 4 \mathrm{~mm}$ made by glycerol; (c) $5 \mathrm{~mm}: 5 \mathrm{~mm}$ made by glycerol; (d) $2.5 \mathrm{~mm}: 5 \mathrm{~mm}$ made by glycerol; (e) $4 \mathrm{~mm}: 5 \mathrm{~mm}$ made by glycerol; (f) $2.5 \mathrm{~mm}$ : $5 \mathrm{~mm}$ made by ethylene glycol.

In conclusion, the diameter and diameter ratio play a decisive role in determining the value of the liquid force, while the liquid volume and surface tension only influence the force value within a local range. For a two-particle system, when the diameter of the upper (lower) particles is the same, a larger diameter ratio will result in a higher liquid force value.

3.4. Rupture Distance. As two particles move, the liquid bridge elongates continuously until it ruptures, and the distance where the bridge breaks is called rupture distance. The results obtained under different experimental conditions are presented in Figure 5.

The bridge made by a larger surface tension liquid gains a higher value for rupture distance as the equal
$2.5 \mathrm{~mm}$ particles, respectively, made by glycerol and ethylene glycol shown in Figure 5. It is obvious that the rupture distance rises up with the increase in liquid volume while falls down with the increase in diameter or diameter ratio. For equal particles, the bridge in two $2.5 \mathrm{~mm}$ equal particles gained the longest rupture distance under the same liquid volume condition. For equal particles, the bridge in $2.5 \mathrm{~mm}: 5 \mathrm{~mm}$ particles has the largest rupture distance value. Meanwhile, the rupture distance in equal particles is much longer than it in unequal particles.

It can be concluded that different from the maximum liquid force, the liquid volume, surface tension, and diameter or diameter ratio all play roles in determining the value of 


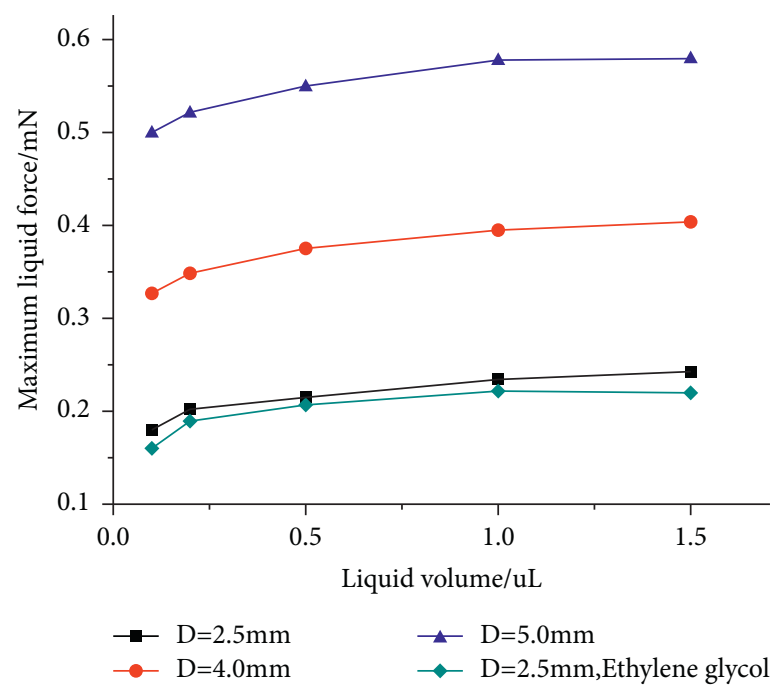

(a)

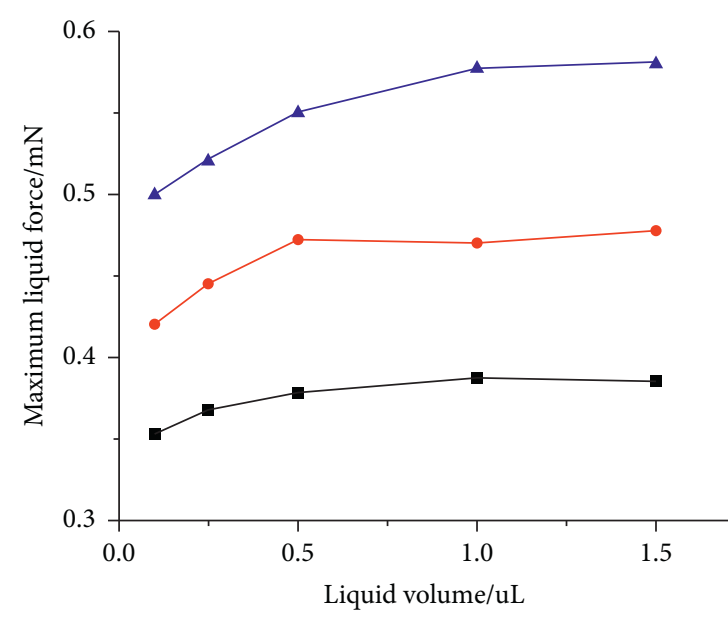

- $\mathrm{D}=2.5 \mathrm{~mm}: 5 \mathrm{~mm}$

$\longrightarrow \mathrm{D}=4 \mathrm{~mm}: 5 \mathrm{~mm}$

$\leadsto \mathrm{D}=5 \mathrm{~mm}: 5 \mathrm{~mm}$

FIgURE 4: The maximum liquid force-liquid volume curves. (a) equal particles; (b) unequal particles.

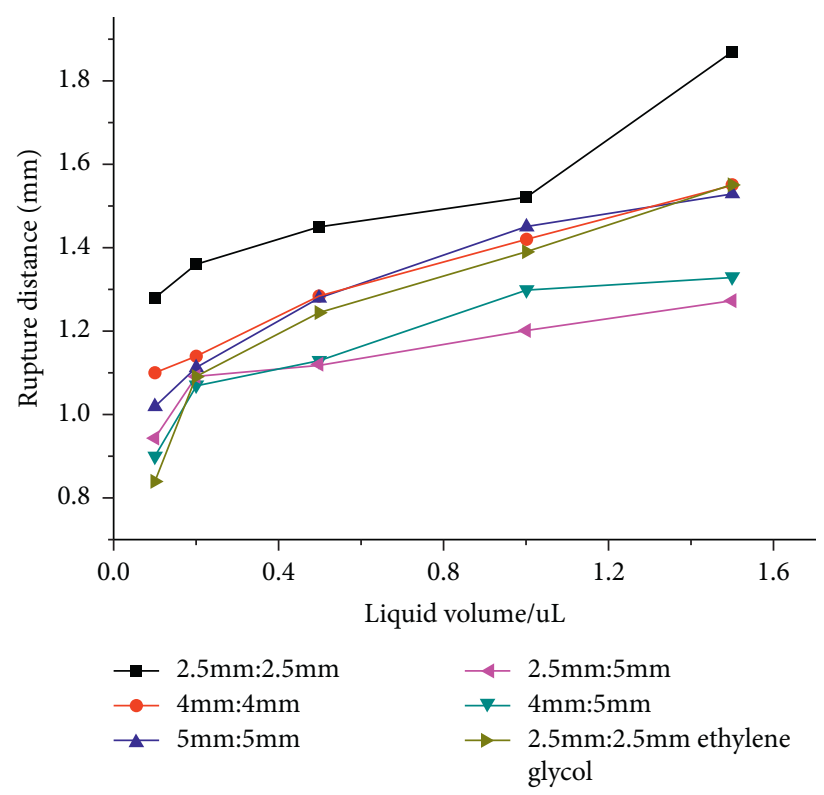

Figure 5: Curve of rupture distance-liquid volume.

rupture distance. The rupture shows a positive correlation with liquid volume and surface tension and a negative correlation with the diameter or diameter ratio.

3.5. Comparison of Liquid Force between Equal and Unequal Spheres. Plotting the maximum liquid force between equal particles and unequal particles in the same graph, Figure 6(a) shows the maximum liquid force of equal and unequal particles system when the diameter of upper particle equals $2.5 \mathrm{~mm}$ and Figure $6(\mathrm{~b})$ shows the diameter of upper particle equals $5 \mathrm{~mm}$.

The red dots with the solid line represent the maximum force between two unequal particles, the blue triangles represent the maximum force between two equal particles whose size is equal to that of the upper particles in the unequal particle system, the black squares represent the maximum force between two equal particles whose size is 


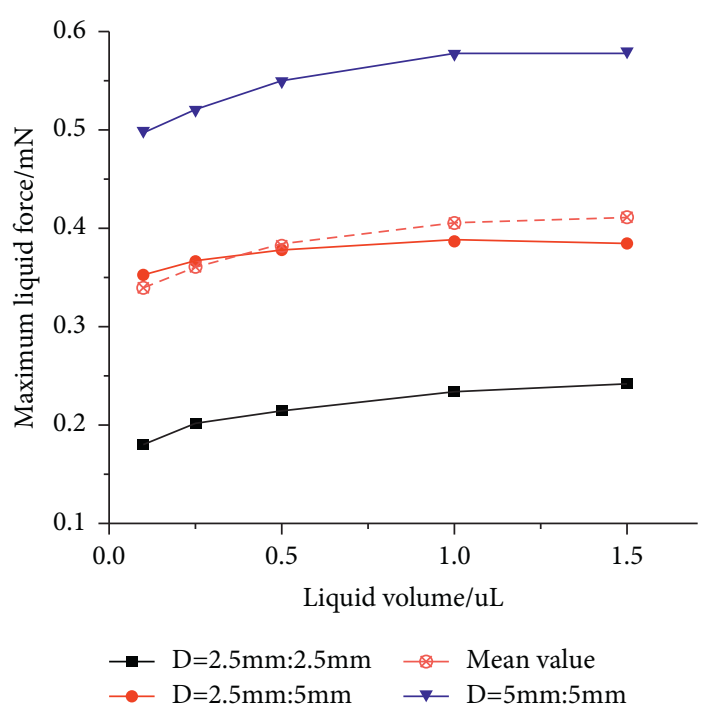

(a)

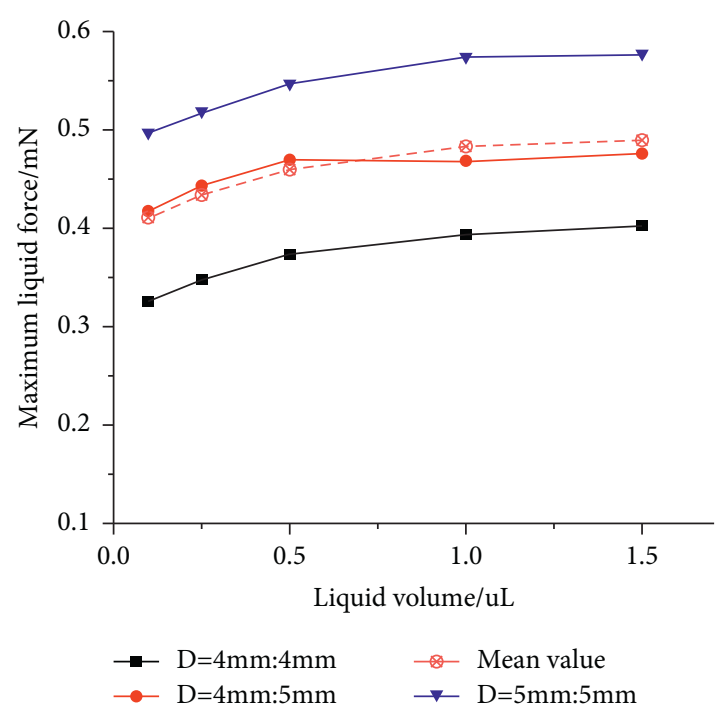

(b)

Figure 6: The maximum liquid force-liquid volume curves: (a) the upper sphere equals $2.5 \mathrm{~mm}$; (b) the upper sphere equals $4 \mathrm{~mm}$.

equal to that of the lower particles, and the red dots with the dashed line represent the mean values of the blue triangles and black squares. It can be seen that, for a system of two unequal particles, the maximum liquid force is lower than the force between equal particles of a larger size and higher than the force in a system of equal particles of a smaller size, and it is about half of the sum of those two forces.

\section{Conclusion}

Using the Nano UTM T150 tension instrument to measure the liquid force between equal and unequal spheres, the influence of sphere diameter, liquid volume, and surface tension on the liquid force and rupture distance was compared. The following conclusions can be made:

(1) The liquid force-displacement curve can be divided into ascent stage, descent stage, and rupture stage, respectively. The difference between the two types of curves is due to the discrepancies between the initial conditions of different test methods. When the maximum force occurred in the zero-separation distance, the experiment starts at a force equilibrium point, though the separation distance may not be zero. When the maximum force appeared at a small but nonzero-separation distance, the experiment starts at a displacement equilibrium point, in which the separation distance is absolute zero, but may exist compressive stress at the top of the bottom particles.

(2) The maximum liquid force and rupture distance both increase with the increase in liquid volume, particle diameter, diameter ratio, and surface tension. The diameter plays a decisive role in determining the value of the maximum liquid force compared with the liquid volume and surface tension, which only influences the force value within a local range. The rupture distance shows a positive correlation with liquid volume and surface tension and a negative correlation with the diameter or diameter ratio.

(3) For a system of unequal spheres, the maximum liquid force is lower than the force between equal particles with a size that equals that of the larger particle in the unequal system and higher than the force between equal particles that equals in size to the smaller particle in the unequal system. The maximum liquid force between unequal particles is about half of the sum of the force between the equal spheres of larger and smaller size in that system.

\section{Data Availability}

The datasets used and/or analyzed during the current study are available from the corresponding author on reasonable request.

\section{Conflicts of Interest}

The authors declare that they have no conflicts of interest.

\section{Acknowledgments}

This study was funded by the National Natural Science Foundation of China (Nos. 51679198 and 12072260) and the Institute of Geotechnical Engineering in Xi'an University of Technology.

\section{References}

[1] R. A. Fisher, "On the capillary forces in an ideal soil; correction of formulae given by W. B. Haines," The Journal of Agricultural Science, vol. 16, no. 3, pp. 492-505, 1926.

[2] T. Gillespie and W. J. Settineri, "The effect of capillary liquid on the force of adhesion between spherical solid particles," Journal of Colloid and Interface Science, vol. 24, no. 2, pp. 199-202, 1967. 
[3] W. C. Clark, J. M. Haynes, and G. Mason, "Liquid bridges between a sphere and a plane," Chemical Engineering Science, vol. 23, no. 7, pp. 810-812, 1968.

[4] V. P. Mehrotra and K. V. S. Sastry, "Pendular bond strength between unequal-sized spherical particles," Powder Technology, vol. 25, no. 2, pp. 203-214, 1980.

[5] F. R. E. De Bisschop and W. J. L. Rigole, "A physical model for liquid capillary bridges between adsorptive solid spheres: the nodoid of plateau," Journal of Colloid and Interface Science, vol. 88, no. 1, pp. 117-128, 1982.

[6] G. Lian, C. Thornton, and M. J. Adams, "A theoretical study of the liquid bridge forces between two rigid spherical bodies," Journal of Colloid and Interface Science, vol. 161, no. 1, pp. 138-147, 1993.

[7] M. J. Matthewson, "Adhesion of spheres by thin liquid films," Philosophical Magazine A, vol. 57, no. 2, pp. 207-216, 1988.

[8] K. Washino, E. L. Chan, H. Midou, T. Tsuji, and T. Tanaka, "Tangential viscous force models for pendular liquid bridge of Newtonian fluid between moving particles," Chemical Engineering Science, vol. 174, pp. 365-373, 2017.

[9] F. Molenkamp and A. H. Nazemi, "Interactions between two rough spheres, water bridge and water vapour," Géotechnique, vol. 53, pp. 255-264, 2013.

[10] D. Pirooz, T. W. Li, K. Pougatch, M. Salcudean, and D. Grecov, "Modeling the evolution and rupture of stretching pendular liquid bridges," Chemical Engineering Science, vol. 65 , pp. 4472-4483, 2010.

[11] Y. Chen, Y. Zhao, H. Gao, and J. Zheng, "Liquid bridge force between two unequal-sized spheres or a sphere and a plane," Particuology, vol. 9, no. 4, pp. 374-380, 2011.

[12] D. Rossetti and S. J. R. Simons, "A microscale investigation of liquid bridges in the spherical agglomeration process," Powder Technology, vol. 130, pp. 49-55, 2013.

[13] D. N. Mazzone, G. I. Tardos, and R. Pfeffer, "The effect of gravity on the shape and strength of a liquid bridge between two spheres," Journal of Colloid and Interface Science, vol. 113, no. 2, pp. 544-556, 1986.

[14] T. P. Farmer and J. C. Bird, "Asymmetric capillary bridges between contacting spheres," Journal of Colloid and Interface Science, vol. 454, pp. 192-199, 2015.

[15] C. Zhang, Z. Liu, and Y. Dong, "Effects of adsorptive water on the rupture of nanoscale liquid bridges," Applied Clay Science, vol. 146, pp. 487-494, 2017.

[16] O. Pitois, P. Moucheront, and X. Chateau, "Rupture energy of a pendular liquid bridge," The European Physical Journal B, vol. 23, no. 1, pp. 79-86, 2001.

[17] O. Pitois, P. Moucheront, and X. Chateau, "Liquid bridge between two moving spheres: an experimental study of viscosity effects," Journal of Colloid and Interface Science, vol. 231, no. 1, pp. 26-31, 2000.

[18] C. D. Willett, M. J. Adams, S. A. Johnson, and J. P. K. Seville, "Capillary bridges between two spherical bodies," Langmuir, vol. 16, no. 24, pp. 9396-9405, 2000.

[19] D. Rossetti, X. Pepin, and S. J. R. Simons, "Rupture energy and wetting behavior of pendular liquid bridges in relation to the spherical agglomeration process," Journal of Colloid and Interface Science, vol. 261, no. 1, pp. 161-169, 2003.

[20] B. Mielniczuk, T. Hueckel, and M. S. E. Youssoufi, "Evaporation-induced evolution of the capillary force between two grains," Granular Matter, vol. 16, no. 5, pp. 815-828, 2014.

[21] M. G. Bozkurt, D. Fratta, and W. J. Likos, "Capillary forces between equally sized moving glass beads: an experimental study," Canadian Geotechnical Journal, vol. 54, no. 9, pp. 1300-1309, 2017.
[22] C. Pu, F. Y. Liu, and Z. Zhang, "Liquid force and profile between two moving sphere particles under different liquid content condition: an experiment study," Shuili Xuebao, vol. 51, pp. 81-91, 2020, in Chinese.

[23] D. Lievano, S. Velankar, and J. J. McCarthy, "The rupture force of liquid bridges in two and three particle systems," Powder Technology, vol. 313, pp. 18-26, 2017.

[24] J. P. Wang, E. Gallo, and B. Francois, "Capillary force and rupture of funicular liquid bridges between three spherical bodies," Powder Technology, vol. 305, pp. 89-98, 2016.

[25] Y. I. Rabinovich, M. S. Esayanur, and B. M. Moudgil, "Capillary forces between two spheres with a fixed volume liquid bridge: theory and experiment," Langmuir, vol. 21, no. 24, pp. 10992-10997, 2005.

[26] H. Kazuyuki, T. Kazuo, and I. Koichi, "The capillary binding force of a liquid bridge," Powder Technology, vol. 10, pp. 231-242, 1974.

[27] F. Soulié, F. Cherblanc, M. S. El Youssoufi, and C. Saix, "Influence of liquid bridges on the mechanical behaviour of polydisperse granular materials," International Journal for Numerical and Analytical Methods in Geomechanics, vol. 30, no. 3, pp. 213-228, 2006. 